\title{
Real-Time Circumferential Mapping Catheter Tracking for Motion Compensation in Atrial Fibrillation Ablation Procedures
}

\author{
Alexander Brost ${ }^{a}$, Felix Bourier ${ }^{b}$, Andreas Wimmer $^{a}$, Martin $\operatorname{Koch}^{a}$, \\ Atilla Kiraly $^{c}$, Rui Liao ${ }^{c}$, Klaus Kurzidim ${ }^{b}$, Joachim Hornegger $^{a, e}$ and \\ Norbert Strobel ${ }^{d}$ \\ ${ }^{a}$ Pattern Recognition Lab, Friedrich-Alexander-University \\ Erlangen-Nuremberg, Erlangen, Germany \\ ${ }^{b}$ Klinik für Herzrhythmusstörungen, Krankenhaus Barmherzige Brüder, \\ Regensburg, Germany \\ ${ }^{c}$ Siemens Corporate Research, Princeton, NJ, USA \\ ${ }^{d}$ Siemens AG, Healthcare Sector, Forchheim, Germany \\ ${ }^{e}$ School in Advanced Optical Technologies (SAOT), Erlangen, Germany
}

\begin{abstract}
Atrial fibrillation (AFib) has been identified as a major cause of stroke. Radiofrequency catheter ablation has become an increasingly important treatment option, especially when drug therapy fails. Navigation under X-ray can be enhanced by using augmented fluoroscopy. It renders overlay images from pre-operative 3-D data sets which are then fused with $\mathrm{X}$-ray images to provide more details about the underlying soft-tissue anatomy. Unfortunately, these fluoroscopic overlay images are compromised by respiratory and cardiac motion. Various methods to deal with motion have been proposed. To meet clinical demands, they have to be fast. Methods providing a processing frame rate of 3 frames-per-second (fps) are considered suitable for interventional electrophysiology catheter procedures if an acquisition frame rate of $2 \mathrm{fps}$ is used. Unfortunately, when working at a processing rate of $3 \mathrm{fps}$, the delay until the actual motion compensated image can be displayed is about $300 \mathrm{~ms}$. By using a novel approach involving a 3-D catheter model, catheter segmentation and a distance transform, we can speed up motion compensation to $20 \mathrm{fps}$ which results in a display delay of only $50 \mathrm{~ms}$ on a standard workstation for medical applications. Our method uses a constrained 2-D/3-D registration to perform catheter tracking, and it obtained a 2-D tracking error of $0.61 \mathrm{~mm}$.
\end{abstract}

Keywords: Ablation Procedures, Catheter Tracking, Electrophysiology, Motion Compensation

\section{MOTIVATION}

Atrial fibrillation (AFib) is the most common heart arrhythmia, and leads to an increased risk of stroke for the patients. Since the first treatment approaches using radio-frequency ablation, this method has now become an accepted treatment option, in particular when drug therapy fails. Catheter ablation procedures are performed in electrophysiology (EP) labs usually equipped with modern C-arm X-ray systems. Augmented fluoroscopy, overlaying 2-D renderings obtained from either CT, MR, or C-arm CT 3-D data sets onto live fluoroscopic images, can facilitate more precise real-time catheter navigation and also reduce radiation. Unfortunately,

Send correspondence to Alexander Brost: Alexander.Brost@cs.fau.de 
catheter navigation under augmented fluoroscopy is compromised by cardiac and respiratory motion. A first approach to tackle this problem by providing a motion compensated overlay was proposed in [1]. It involved tracking of commonly used circumferential mapping catheters. As AFib therapy takes place in the vicinity of the circumferential mapping catheter, tracking the catheter can be assumed to capture the motion of the relevant treatment region correctly if the device has been firmly positioned. Fortunately, we can count on the physicians to provide a stable wall contact, as it is in their best interest. Otherwise complete isolation of the pulmonary veins (PVs) may fail due to undetected residual PV-atrial electrical connections. The initially proposed method generated a 3-D model of the catheter and applied an unconstrained 2-D/3-D registration to register the catheter model to biplane fluoroscopy images. This work was extended regarding robustness using a learning-based catheter segmentation. The approach was also modified to work on monoplane systems as well [2]. The latest extension of this work can be found in [2]. This approach does not require simultaneously biplane fluoroscopy but instead uses a constrained 2-D/3-D registration to achieve motion compensation if only monoplane acquisitions are used at a biplane system. We extended this method to achieve real-time capability, even outperforming other motion compensation approaches [3].

\section{METHODS}

The proposed method comprises three main steps. First, a 3-D model of the mapping catheter is generated. Second, the catheter is segmented in 2-D X-ray images using a classifier. The segmentation result is refined next. The main difference to previous approaches is that our method does not use the a thinning algorithm. Finally, the catheter is tracked relying on 2$\mathrm{D} / 3$-D registration of the 3-D catheter model to the 2-D X-ray images. The resulting motion information can be used to adjust the fluoroscopic overlay accordingly. Below, these steps are explained in more detail.

Model Generation In the first step of our method, a 3-D model of the circumferential mapping catheter is generated, as proposed in $[1,4,5]$. A single biplane $\mathrm{X}$-ray acquisition is required to manually initialize the model generation step. To this end, 2-D points, $\mathbf{p}_{\mathrm{A}}, \mathbf{p}_{\mathrm{B}} \in \mathbb{R}^{2}$, are selected on the elliptically-shaped part of the catheter in each imaging plane. The imaging planes are indexed by their respective notation of plane $A$ and plane $B$. Two-dimensional ellipses in the imaging planes are then fitted to these points [6]. The implicit ellipse parameters are given as $\mathbf{C}_{\mathrm{A}}, \mathbf{C}_{\mathrm{B}} \in \mathbb{R}^{3 \times 3}$. Two 3-D cones are spanned from the cameras' optical centers to the ellipses on the imaging planes, using the projection matrices $\mathbf{P}_{\mathrm{A}}, \mathbf{P}_{\mathrm{B}} \in \mathbb{R}^{3 \times 4}$, resulting in

$$
\begin{aligned}
& \mathbf{Q}_{\mathrm{A}}=\mathbf{P}_{\mathrm{A}}^{T} \mathbf{C}_{\mathrm{A}} \mathbf{P}_{\mathrm{A}} \\
& \mathbf{Q}_{\mathrm{B}}=\mathbf{P}_{\mathrm{B}}^{T} \mathbf{C}_{\mathrm{B}} \mathbf{P}_{\mathrm{B}} .
\end{aligned}
$$

Every intersection of a plane with one of the cones yields a valid solution of an ellipse in 3-D when projected onto the respective imaging plane. Two possible solutions for the reconstructed ellipse in 3-D space can be found [1]. In our application, we utilize prior knowledge about the pseudo-circular shape of the circumferential mapping catheter and select the result that is more circular, because the circumferential mapping catheter inserted into a pulmonary vein resembles a circle more closely than an ellipse in normal human anatomy. The circularity is determined by

$$
\kappa=|\phi-\psi|
$$

with the axes $\phi$ and $\psi$ of an ellipse. To obtain the more circular solution, the ellipse with the smaller value for $\kappa$ is used. The computation is done by

$$
\hat{\chi}=\arg \min _{\chi \in\{1,2\}}\left|\phi_{\chi}-\psi_{\chi}\right|
$$


with $\chi \in\{1,2\}$ denoting either the first or the second possible solution. The accepted 3-D model corresponding to solution $\hat{\chi}$ is denoted as $\mathbf{m}_{i} \in \mathbf{R}^{4}$ in homogeneous coordinates as $\mathbf{m}_{i}=\left(m_{i, x}, m_{i, y}, m_{i, z}, 1\right)^{T} \in \mathbb{R}^{4}$ with $i \in\{1,2, \ldots, N\}$ and $N$ the number of model points. This step is performed only once.

Image Processing Once the 3-D model of the catheter has been generated, only monoplane fluoroscopic imaging is needed for the remaining tasks of motion estimation. Compared to the monoplane method in [2], the newly proposed method does not require manual re-initialization of the catheter model when $\mathrm{C}$-arm angulation changes, minimizing user interaction during interventions. The catheter segmentation method not only has to be reliable, but it needs to be fast as well. Speed is necessary to ensure that the catheter can be tracked in real-time at the frame rate set at the X-ray acquisition system. We found that a combination of Haar-like features and a cascade of boosted classifiers satisfied both requirements, see Fig. 1 (b) for an example. For evaluation, the classifier cascade was trained on a leave-one-out basis. As the segmentation is trained for the whole catheter, previous methods [2] used a thinning algorithm as proposed in [7] to calculate the centerline of the catheter. Afterwards, a distance transform was used to calculate the distance of every pixel to the closest centerline pixel. There are several drawbacks to this method. First of all, the runtime of the thinning process is not linear with the pixels but with the actual pixels that are classified as catheter. To fix this would require a better segmentation, which is also more time consuming. Second, a single misclassified pixel has a rather high impact, see Fig. 1 (c). As a solution to this problem we propose to apply the distance transform to the pixels classified as catheter and not to the pixels identified as background. Further, we do not make use of a thinning algorithm. By doing so, the distance transform is only calculated for those pixels segmented as catheter (the white pixels), and the highest values of the distance transform are found along the centerline of the catheter, see Fig. 1 (d). The distance transform of the catheter segmentation is denoted as $\mathbf{I}_{\mathrm{DT}}$.

Tracking by Constrained Registration In the third step, catheter tracking is performed by rigid registration of the 3-D catheter model using the distance map derived in the previous step. The pre-processed image $\mathbf{I}_{\mathrm{DT}}$ is considered as cost function. It has a maximum at the segmented catheter position. The 3-D catheter model is moved in 3-D parallel to the imaging plane and forward projected onto the imaging plane. To carry out a translation parallel to the imaging plane, the viewing direction $\mathbf{n} \in \mathbb{R}^{3}$ with $\|\mathbf{n}\|_{2}=1$ is obtained from the projection matrix $\mathbf{P} \in \mathbb{R}^{3 \times 4}$ for either imaging plane $A$ or imaging plane $B$. As the viewing direction is perpendicular to all vectors that are parallel to the imaging plane, two spanning vectors, defining the search plane, need to be calculated. To do so, an arbitrary vector $\mathbf{w} \in \mathbf{R}^{3}$ with $\|\mathbf{w}\|_{2}=1$ and $\mathbf{n}^{T} \mathbf{w} \neq 0$ is chosen. The two spanning vectors $\mathbf{u}, \mathbf{v} \in \mathbb{R}^{3}$ of the search plane are then computed by

$$
\begin{aligned}
\mathbf{u} & =\mathbf{n} \times \mathbf{w} \\
\mathbf{v} & =\mathbf{n} \times \mathbf{u}
\end{aligned}
$$

with $\|\mathbf{u}\|_{2}=1$ and $\|\mathbf{v}\|_{2}=1$, and $\mathbf{u}=\left(u_{x}, u_{y}, u_{z}\right)^{T}$ and $\mathbf{v}=\left(v_{x}, v_{y}, v_{z}\right)^{T}$. The vectors $\mathbf{u}$ and $\mathbf{v}$ are dependent on the choice of $\mathbf{w}$. For a differently chosen vector $\mathbf{w}$, the vectors $\mathbf{u}$ and $\mathbf{V}$ might be different, but they still span the same search plane. Any point on this plane can be represented by a linear combination of the these two vectors. Thus, moving a point $q \in \mathbb{R}^{3}$ in parallel to the imaging plane is achieved by using the following translation matrix

$$
\mathbf{T}(\lambda, \mu)=\left(\begin{array}{cccc}
1 & 0 & 0 & \lambda u_{x}+\mu v_{x} \\
0 & 1 & 0 & \lambda u_{y}+\mu v_{y} \\
0 & 0 & 1 & \lambda u_{z}+\mu v_{z} \\
0 & 0 & 0 & 1
\end{array}\right)
$$




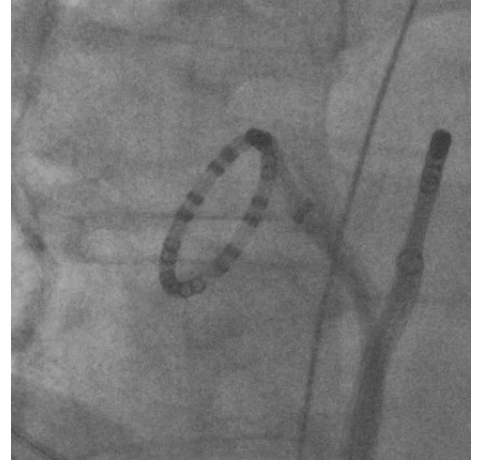

(a)

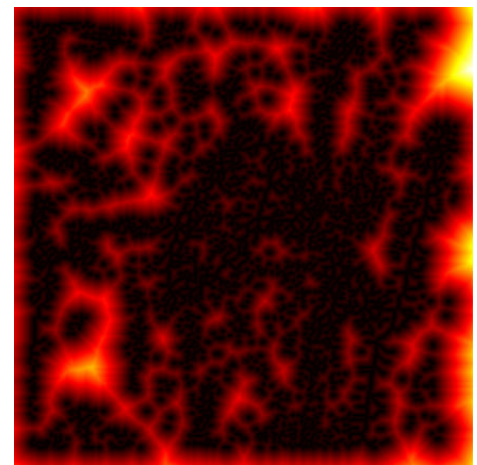

(c)

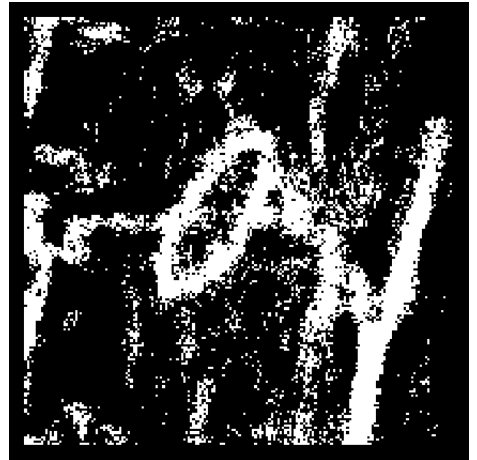

(b)

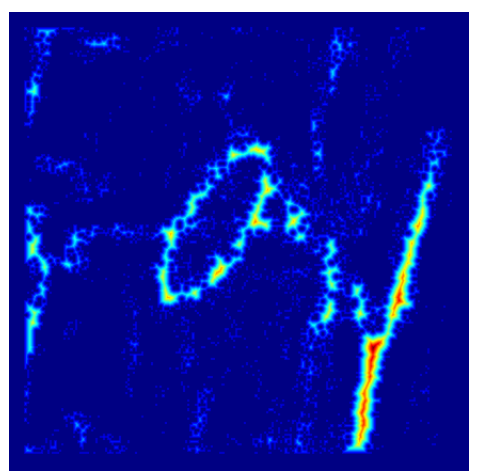

(d)

Figure 1: (a) Cropped fluorscopic image around the region-of-interest, determined from manual initialization or a previous frame. (b) Segmentation using a boosted classifier cascade. The black pixels in the image represent pixels that are identified as background, whereas the white pixels are classified as catheter. (c) Distance transform for all black pixels after applying skeletonization to the segmentation. The dark color indicates a small distance to the segmented catheter. The colors over red to yellow indicate a higher distance to pixels classified as catheter. (d) Distance transform obtained by calculating how far pixels classified as catheter (white pixels) are away from pixels classified as background (black pixels). Dark blue indicates a large distance to the centerline of the catheter. The colors over green yellow to red indicate a closer distance to the catheter centerline. This image is denoted as $\mathbf{I}_{\mathrm{DT}}$.

with the translated point $\mathbf{q}^{\star} \in \mathbb{R}^{3}$ and the amount of translation defined by $\lambda, \mu \in \mathbb{R}$. The objective function for the constrained registration is then defined by using the values of the distance transformed image $\mathbf{I}_{\mathrm{DT}}$ as cost function. The sum over all projected model points into the image $\mathbf{I}_{\mathrm{DT}}$ is maximized. Instead of using a minimization problem as proposed in [2], the maximum of the cost function defines the best location. The maximization is given by

$$
\hat{\lambda}, \hat{\mu}=\arg \max _{\lambda, \mu} \sum_{i} \mathbf{I}_{\mathrm{DT}}\left(\mathbf{P} \cdot \mathbf{T}(\lambda, \mu) \cdot \mathbf{m}_{i}\right) .
$$

The parameters $\hat{\lambda}$ and $\hat{\mu}$ are found by a nearest-neighbor search. Applying $\mathbf{T}(\hat{\lambda}, \hat{\mu})$ then moves the 3-D volumetric data set in sync with the live fluoroscopic images. This way, we can achieve a 3-D motion compensation for mono-plane fluoroscopic images.

\section{EVALUATION AND RESULTS}

For the evaluation of the proposed method, 13 clinical biplane sequences were available. Biplane sequences were required to calculate an estimate for the 3-D error of our motion compensation approach. The 2-D tracking error was calculated as the average distance between the 


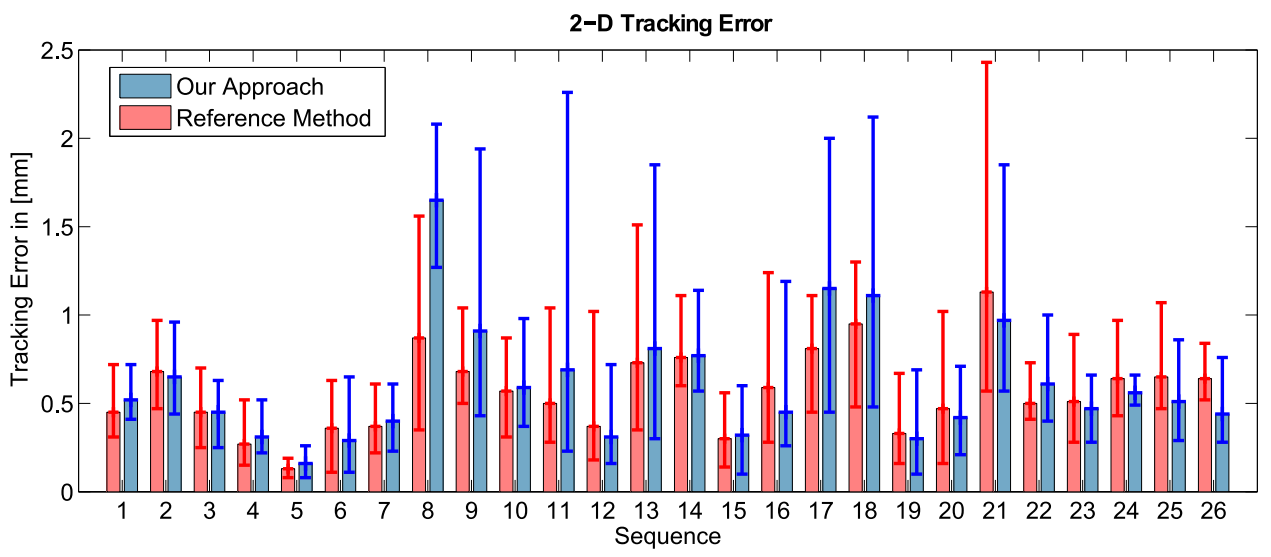

(a)

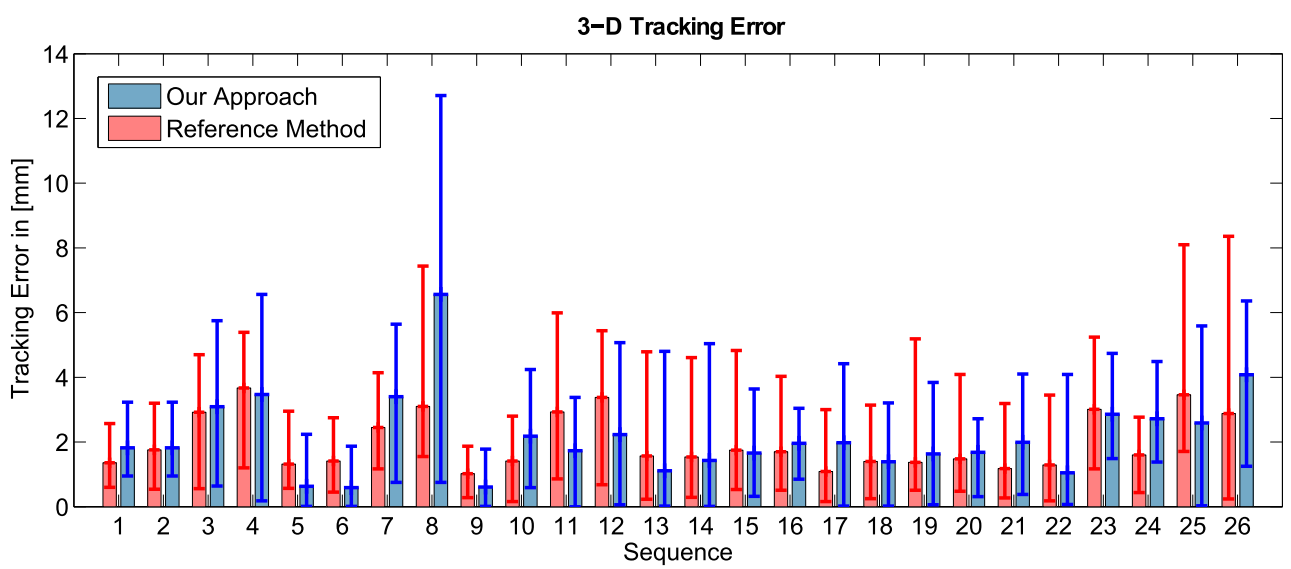

(b)

Figure 2: Tracking results of our new method in comparison to the reference method [2]. (a) Twodimensional tracking error for all 26 sequences. For each sequence, the average, minimum, and maximum for the tracking error as well as the observed motion is given. On average, we observed for our new method an overall mean error of $0.61 \mathrm{~mm}$, an overall minimum error of $0.08 \mathrm{~mm}$, and an overall maximum error of $2.26 \mathrm{~mm}$. The reference method yielded an overall mean of (b) Three-dimensional tracking error for all 26 sequences. We found an overall mean error of $2.16 \mathrm{~mm}$, an overall minimum error of $0.01 \mathrm{~mm}$, and an overall maximum error of $12.71 \mathrm{~mm}$.

motion-compensated catheter model and the manually segmented mapping catheter. Manual segmentation was supervised by a cardiologist. This distance was averaged over all frames of a particular sequence to arrive at an overall tracking error for each sequence. Our proposed method achieved a 2-D tracking error of $0.61 \mathrm{~mm} \pm 0.45 \mathrm{~mm}$. The 3-D tracking error was evaluated by comparing the 3-D motion vector obtained from our constrained motion compensation approach to a 3-D motion vector obtained from the biplane sequences. The 3-D error turned out to be $2.10 \mathrm{~mm} \pm 1.26 \mathrm{~mm}$. The results are given in Fig. 2 .

\section{DISCUSSION AND CONCLUSIONS}

We developed a method for respiratory and cardiac motion compensation for use in radiofrequency catheter ablation of atrial fibrillation that is capable of real-time processing. It outperforms a different approach for motion compensation [3] and its accuracy. Although the accuracy is slightly lower than other methods [2], the speed-up might be the key for its application as this 
method does not put any restrictions on the procedure regarding the acquisition frame rate. Excluding the skeletonization from the image processing pipeline has two effects. First, the processing speed increases, but at the same time, the method becomes less accurate [2]. The difference between our fast version and [2] is at most $0.74 \mathrm{~mm}$ or $\sim 4$ pixels in the fluoroscopic image. The presented method appears to have a rather high 3-D error, however this is because we try to estimate 3-D motion from a single X-ray view. It is possible to reduce the 3-D error by resorting to simultaneous biplane X-ray imaging [1], but this increases the X-ray dose for patient and physician. We consider our method as another step towards a fully integrated software guidance application for electrophysiology procedures, especially atrial fibrillation ablation procedures, that accommodates physicians' needs as much as possible. Our implementation of the reference method [2] was found to be able to process at least 10 frames-per-second (fps). Our new approach achieved a minimum frame rate of $20 \mathrm{fps}$.

\section{Acknowledgements}

This work has been supported by the German Federal Ministry of Education and Research (BMBF), project grant No. 01EX1012E, in the context of the initiative Spitzencluster Medical Valley - Europäische Metropolregion Nürnberg. Additional funding was provided by Siemens AG, Healthcare Sector.

\section{References}

[1] A. Brost, R. Liao, J. Hornegger, and N. Strobel, "3-D Respiratory Motion Compensation during EP Procedures by Image-Based 3-D Lasso Catheter Model Generation and Tracking," in MICCAI 2009, September 20 - 24, London, UK, LNCS 5761, 394-401, Springer Berlin / Heidelberg (2009).

[2] A. Brost, A. Wimmer, R. Liao, J. Hornegger, and N. Strobel, "Constrained 2-D/3-D Registration for Motion Compensation in AFib Ablation Procedures," in IPCAI 2011, Berlin, Germany, LNCS 6689, 133-144, Springer Berlin / Heidelberg (2011).

[3] Y. Ma, A. King, N. Gogin, C. Rinaldi, J. Gill, R. Razavi, and K. Rhode, "Real-Time Respiratory Motion Correction for Cardiac Electrophysiology Procedures Using Image-Based Coronary Sinus Catheter Tracking," in MICCAI 2010, September 20 - 24, Bejing, China, LNCS 6361, 391-399, Springer Berlin / Heidelberg (2010).

[4] A. Brost, R. Liao, N. Strobel, and J. Hornegger, "Respiratory motion compensation by model-based catheter tracking during EP procedures," Medical Image Analysis 14(5), 695 - 706 (2010). Special Issue on the 12th International Conference on Medical Image Computing and Computer-Assisted Intervention (MICCAI) 2009.

[5] A. Brost, A. Wimmer, R. Liao, J. Hornegger, and N. Strobel, "Catheter Tracking: FilterBased vs. Learning-Based," in Pattern Recognition, M. Goesele, S. Roth, A. Kuijper, B. Schiele, and K. Schindler, Eds., Lecture Notes in Computer Science 6376, 293-302, Springer Berlin / Heidelberg (2010).

[6] R. Halir and J. Flusser, "Numerically Stable Direct Least Squares Fitting Of Ellipses," in WSCG (International Conferences in Central Europe on Computer Graphics, Visualization and Computer Vision), February 9 - 13, 1998, University of West Bohemia, Campus Bory, Plzen - Bory, Czech Republic, 253-257 (1998).

[7] J. Cychosz, "Efficient Binary Image Thinning using Neighborhood Maps," in Graphics Gems IV, 465-473, Academic Press Professional, Inc., San Diego, CA, USA (1994). 